\title{
Editorial
}

\section{Applications of Magnetorheological Technology to Semiactive Vibration Control Systems}

\author{
Miao Yu, ${ }^{1}$ Seung-Bok Choi, ${ }^{2}$ Norman M. Wereley, ${ }^{3}$ Weihua Li, ${ }^{4}$ \\ Jeong-Hoi Koo, ${ }^{5}$ and Xinchun Guan ${ }^{6}$ \\ ${ }^{1}$ Department of Optoelectronics, Chongqing University, Chongqing 400044, China \\ ${ }^{2}$ Smart Structures and Systems Laboratory, Department of Mechanical Engineering, Inha University, \\ Incheon 402-751, Republic of Korea \\ ${ }^{3}$ Department of Aerospace Engineering, University of Maryland, College Park, MD 20742, USA \\ ${ }^{4}$ School of Mechanical, Materials and Mechatronic Engineering, University of Wollongong, Wollongong, NSW2522, Australia \\ ${ }^{5}$ Department of Mechanical and Manufacturing University, Miami University, Oxford, OH 45056, USA \\ ${ }^{6}$ School of Civil Engineering, Harbin Institute of Technology, Harbin, China \\ Correspondence should be addressed to Miao Yu; yumiao@cqu.edu.cn
}

Received 12 May 2015; Accepted 12 May 2015

Copyright ( 2015 Miao Yu et al. This is an open access article distributed under the Creative Commons Attribution License, which permits unrestricted use, distribution, and reproduction in any medium, provided the original work is properly cited.

Semiactive vibration control systems possess both the simplicity and inherent reliability of passive systems while maintaining the adaptability of active vibration control systems integrated with an appropriate control algorithm. One of the recent and promising technologies in semiactive vibration control is to utilize magnetorheological (MR) fluids (MRFs) [1] and MR elastomers (MREs) [2]. The well-known remarkable feature of MR materials is the continuous controllability of stiffness and damping properties under external magnetic field. The unique controllable characteristic of MR materials has catalyzed comprehensive research and development focusing on vibration control systems including vehicle dampers, engine mounts, adaptive tuned vibration absorber, and seismic bearings [3-10]. The major goal of this special issue is to provide recent research in applications utilizing MR materials. In particular, the special issue focuses on the MR techniques that deal with configuration design, dynamic modeling, and semiactive controller implementation along with simulation or/and experimental works. Therefore, the papers published in this special issue will be innovative references for the development of more advanced application devices or systems utilizing MR materials.

In this special issue, all the papers have been accepted and published after strict review process by the worldwide experts on the MR materials technology. The published papers cover characteristic analysis and design of the devices, new modeling approaches, and control algorithms used in vibration control systems. A brief summary of each application proposed in the published papers is given as follows.

J. C. Tudon-Martinez and R. Morales-Menendez proposed a new adaptive vibration control system (AVCS) based on the linear parameter-varying (LPV) control theory to reduce the lack of damping force of MR damper particularly caused by oil leakages and compared the estimation error of the fault with the model of damping force in which the static equations are used. Y. Zhang et al. presented an MR damping turning tool based on the squeeze-mode to improve the vibration resistance of the tool system on the lathe in which the parameters of MR damper were chosen by the 3D finite element simulations. The effectiveness of MR damping turning tool was verified by undertaking the chattering suppressive experiment. A. F. Jahromi et al. proposed a frequency dependent MR damper model where the viscosity of MR damper was modeled by exponential and Gaussian functions. They experimentally investigated the accuracy and consistency of the model in force simulation. $\mathrm{G}$. Yu et al. developed a self-decoupling magnetorheological damper and established a theoretical model based on 
conservation of energy and the constraint equation. They also explored the influence of temperature changes on the damper performance. T. Komatsuzaki and Y. Iwata applied a frequency-tunable dynamic absorber which was fabricated with MRE to the vibration control of a one-DOF structure, controlled with a real-time stiffness switching algorithm. They compared the damping performance with the passivetype absorber. X. P. Do et al. developed a new direct adaptive fuzzy controller based on a model of interval type 2 fuzzy and $H^{\infty}$ tracking techniques and the effectiveness of controller was verified by investigating the field-dependent damping force and the stable robustness of MR damper system. Z. Yulin and Z. Xiuyang proposed the decomposition least mean square (LMS) algorithm and reconstruction LMS algorithm based on Wavelet. The noise reduction performance of the proposed algorithms was compared with traditional LMS algorithm by application to MRE noise control system.

\section{Acknowledgments}

We very much appreciate all the authors' excellent contributions to this special issue and the reviewers' efforts to strictly examine the submitted papers.

Miao Yu Seung-Bok Choi Norman M. Wereley Weihua Li Jeong-Hoi Koo Xinchun Guan
[8] H. Mansour, S. Arzanpour, M. F. Golnaraghi, and A. M. Parameswaran, "Semi-active engine mount design using auxiliary magneto-rheological fluid compliance chamber," Vehicle System Dynamics, vol. 49, no. 3, pp. 449-462, 2011.

[9] Y. Zhou, W. H. Li, and M. N. S. Hadi, "Performance comparison between an MRF damper and an MRE isolator incorporated with a building structure," Applied Mechanics and Materials, vol. 37-38, pp. 862-865, 2010.

[10] S.-H. Eem, H.-J. Jung, and J.-H. Koo, “Application of MR elastomers for improving seismic protection of base-isolated structures," IEEE Transactions on Magnetics, vol. 47, no. 10, pp. 2901-2904, 2011.

\section{References}

[1] M. Yu, X. M. Dong, S. B. Choi, and C. R. Liao, "Human simulated intelligent control of vehicle suspension system with MR dampers," Journal of Sound and Vibration, vol. 319, no. 3-5, pp. 753-767, 2009.

[2] Y. C. Li, J. C. Li, T. F. Tian, and W. H. Li, "A highly adjustable magnetorheological elastomer base isolator for applications of real-time adaptive control," Smart Materials and Structures, vol. 22, no. 9, Article ID 095020, 2013.

[3] P. F. Guo, X. C. Guan, and J. P. Ou, "Physical modeling and design method of the hysteretic behavior of magnetorheological dampers," Journal of Intelligent Material Systems and Structures, vol. 25, no. 6, pp. 680-696, 2014.

[4] M. Yu, B. X. Ju, J. Fu, X. Liu, and Q. Yang, "Influence of composition of carbonyl iron particles on dynamic mechanical properties of magnetorheological elastomers," Journal of Magnetism and Magnetic Materials, vol. 324, no. 13, pp. 2147-2152, 2012.

[5] S.-B. Choi, M.-H. Nam, and B.-K. Lee, "Vibration control of a MR seat damper for commercial vehicles," Journal of Intelligent Material Systems and Structures, vol. 11, no. 12, pp. 936-944, 2001.

[6] M. Yu, C. R. Liao, W. M. Chen, and S. L. Huang, "Study on MR semi-active suspension system and its road testing," Journal of Intelligent Material Systems and Structures, vol. 17, no. 8-9, pp. 801-806, 2006.

[7] Y.-T. Choi and N. M. Wereley, "Semi-active magnetorheological refueling probe systems for aerial refueling events," Smart Materials and Structures, vol. 22, no. 9, Article ID 092001, 2013. 

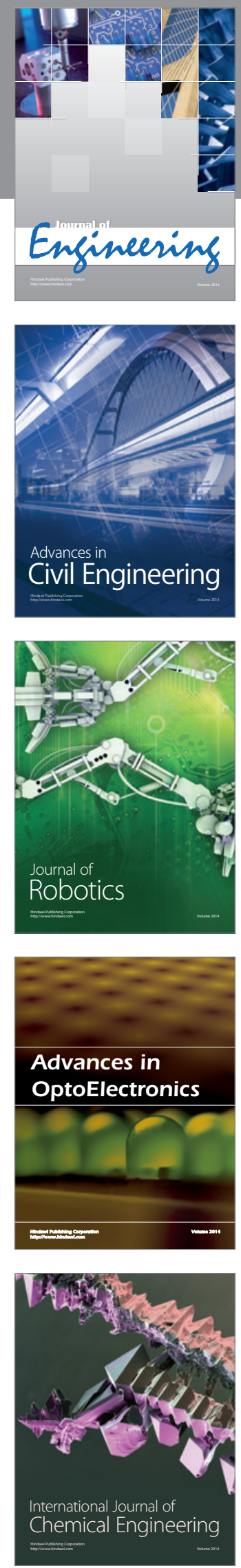

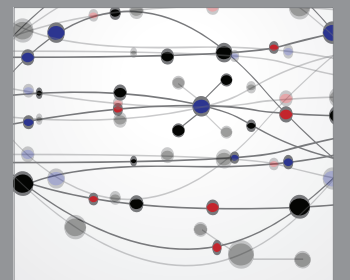

The Scientific World Journal
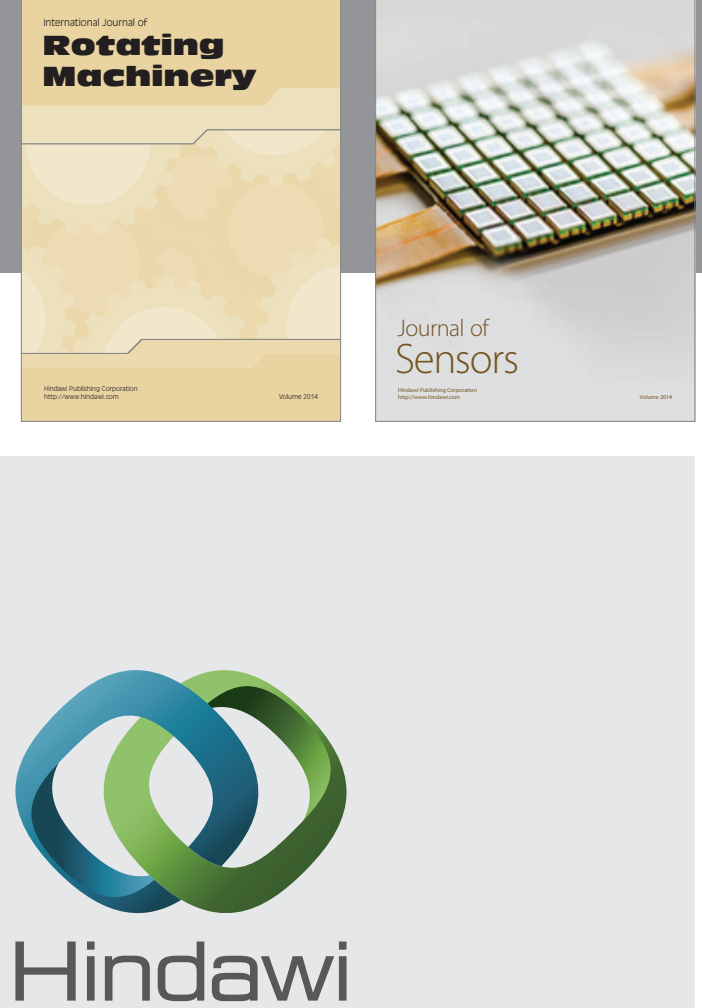

Submit your manuscripts at http://www.hindawi.com
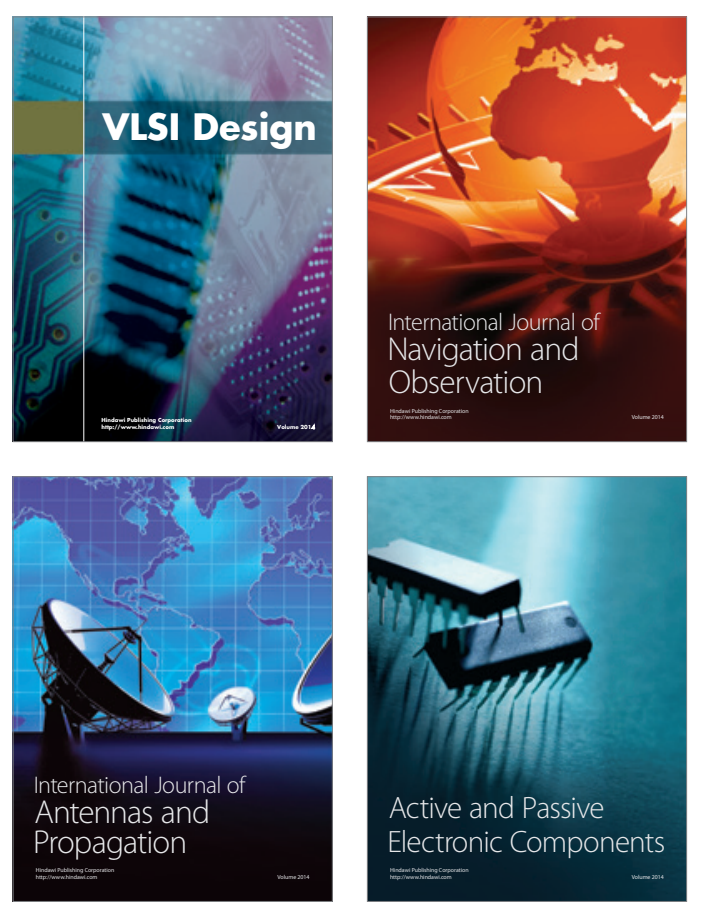
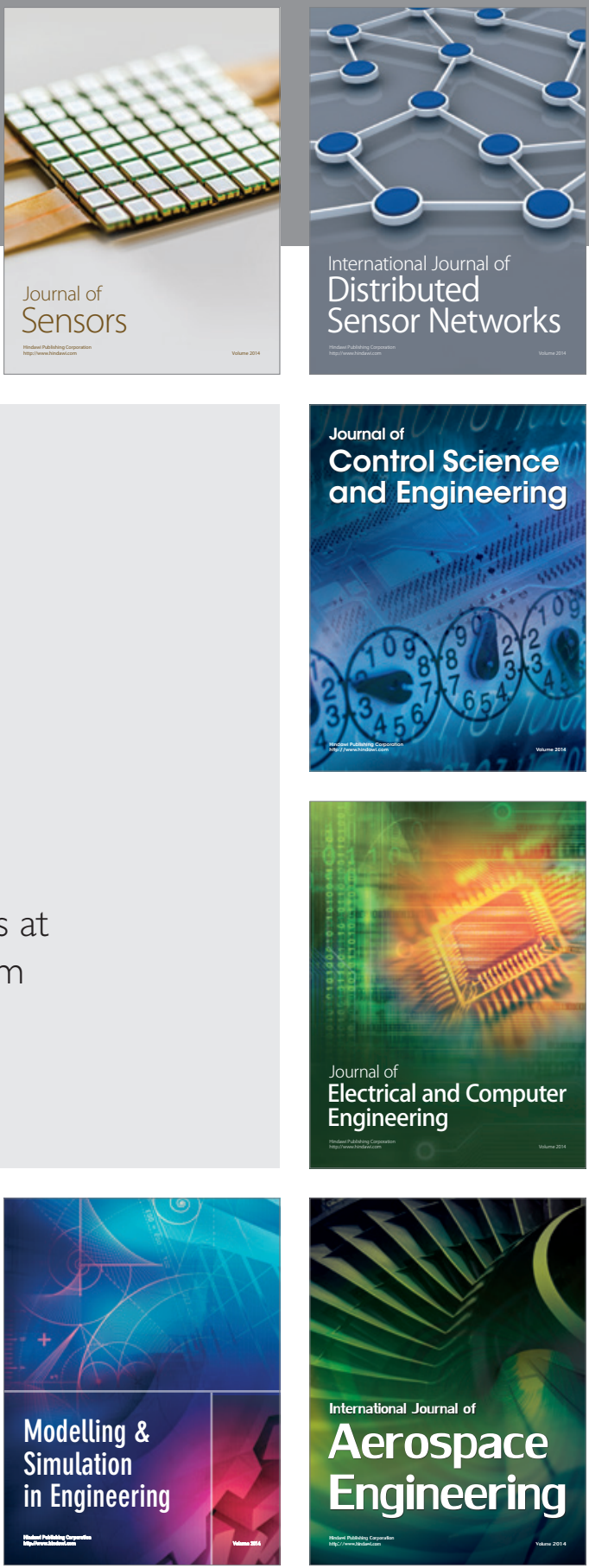

Journal of

Control Science

and Engineering
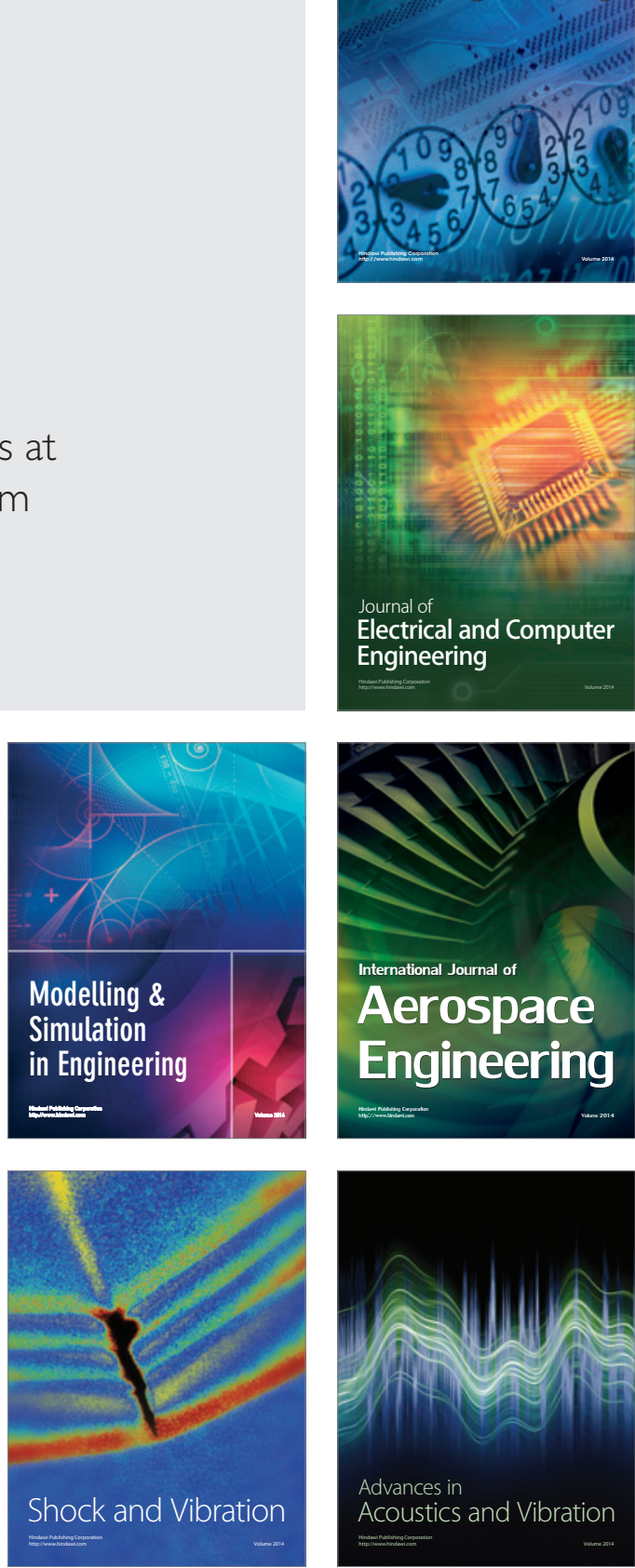